\title{
An operational test for distinguishing between complicated and chaotic behavior in deterministic systems
}

\author{
M. Johnson * J. Habyarimana \\ Department of Mechanical Engineering, Massachusetts Institute of Technology, Room 3-264, 77 Massachusetts Ave., Cambridge, \\ MA 01139, USA
}

Received 29 April 1997; received in revised form 5 December 1997; accepted 15 January 1998

Communicated by C.K.R.T. Jones

\begin{abstract}
While various definitions of chaotic processes have been proposed, there is not yet an established operational criterion for computing whether a given process is chaotic. We here use a diffusional measure to characterize whether a given deterministic process and domain is chaotic (operationally defined as exhibiting stochastic behavior). This technique introduces an additional coordinate linked to the process to be examined. By then determining the growth of the second moment of orbit trajectories in this added direction, it can be determined whether the process is chaotic. It is demonstrated that two other commonly used measures of chaos, a positive Lyapunov exponent or the autocorrelation coefficient dropping to zero, fail in certain cases to detect chaotic processes, but this new proposed test works in all the cases examined. Copyright $\odot 1998$ Elsevier Science B.V.
\end{abstract}

PACS: 05.45

Keywords: Chaos; Diffusion; Lyapunov; Stochastic

\section{Introduction}

Various definitions have been proposed to characterize chaos. Devaney [1] defined these as processes that (i) have a sensitive dependence on initial conditions, (ii) are topologically transitive and (iii) have periodic points that are dense in phase space; said differently, these processes are unpredictable, indecomposable (ergodic) and have an element of regularity. It has recently been shown that the first condition (sensitivity to initial conditions) is a necessary consequency of the second and third conditions [2-4]. Other investigators [5] have defined chaos as

\footnotetext{
*Corresponding author. Tel.: 617-253-7604; fax: 617-2588559; e-mail: markj@mit.edu.
}

a process (i) having infinitely many periodic points and (ii) whose trajectories in phase space may get arbitrarily close to any given point in phase space and yet have no tendency to stay close (non-periodic flow). In this paper, we accept Devaney's definition.

While chaotic processes have been identified, and to some extent characterized, an operational definition or testable criterion is lacking. A distinction has been drawn between low-order chaotic systems and highorder chaotic or "random" processes [6]. We here consider the distinction between chaotic systems (either low or high order) and those systems not exhibiting stochastic behavior (non-chaotic systems).

Merely complexity or anharmonicity is not guaranteed to be chaotic (e.g. the Fermi-Plasta-Ulam problem [7]). A frequently used criterion for chaos is 
the existence of a positive Lyapunov exponent. However, there are a number of difficulties associated with the computation of the Lyapunov exponents including the changing of the principal axes that require frequent renormalization [8] and the boundedness of trajectories that prevent the unbounded exponential growth of a disturbance. It has also not been demonstrated that a chaotic process necessarily exhibits a positive Lyapunov exponent. A second operational definition of chaos that has occasionally been used is the decrease of the autocorrelation function to zero [9] between the original state of the system and the state of the system after $N$ steps (or after time $t$ )

We here look to introduce a new operational test for determining whether a given process is chaotic. We will use this test on a number of well-known chaotic processes and show that in all cases, it correctly identifies chaotic behavior. We will also show that the currently used criteria for chaos (positive Lyapunov exponent and decrease of the autocorrelation coefficient to zero) fail in several cases.

\section{Use of Taylor dispersion to investigate chaotic processes}

Of the different characteristics of chaotic processes that have been identified, perhaps the most universal one is the random-like nature of the processes; this is true for both low-order and high-order chaotic processes. Random processes exhibit diffusion, and many investigators (e.g. [10-12]) have shown that chaotic processes demonstrate diffusion behavior. However, it is difficult in general to determine if a particular chaotic processes is diffusive. With any diffusive process, the geometry of the system will have a strong effect on the dispersion that is difficult to predict $a$ priori without significant analysis. Chaotic mappings are such that, in most cases, the orbits visit most regions of the strange attractor in the first few steps of the process. Since characterization of the dispersion necessarily involves the geometry of the strange attractor, it is difficult to ascertain whether or not dispersion that occurs is consistent with that of diffusive motion.
These difficulties are not unique to chaotic processes. Measurements of the diffusion coefficient of molecular substances are strongly influenced by the system geometry (in this case, the boundary conditions). A procedure that has been used to overcome this difficulty is to take advantage of the Taylor dispersion phenomenon [13]. Taylor [14] made the observation that, in a convective process, diffusion in the cross-sectional plane leads to diffusive-like behavior in the flowwise direction. Specifically, for Poiseuille flow in a circular tube of radius $a$ with mean axial velocity $\bar{V}$, a passive tracer with a molecular diffusion coefficient of $D$ will exhibit diffusive-like behavior in the axial direction (about the mean axial location $\bar{V} t$, where $t$ is time) with an effective diffusion coefficient of

$D_{\text {eff }}=\frac{a^{2} \tilde{V}^{2}}{48 D}$.

The advantage of measuring the dispersion coefficient in the axial direction to determine the molecular diffusion coefficient is that, since a tube can be made very long, boundary conditions in the axial direction become unimportant, and thus $D_{\text {eff }}$ can be easily determined as $1 / 2 \mathrm{~d} \sigma^{2} / \mathrm{d} t$ where $\sigma^{2}$ is the second moment of the tracer about the mean axial position. Non-diffusive processes (e.g. simple Poiseuille flow in a tube without diffusion) show a different character with $\sigma^{2}$ varying as $t^{2}$ (or $N^{2}$ for a discrete process where $N$ is the number of steps). ${ }^{1}$

We here use a similar scheme to investigate chaotic mappings. We consider a general mapping such that

$\hat{x}_{N+1}=g\left(\hat{x}_{N}\right)$,

where $\hat{x} \in \mathbb{R}^{n}$. We introduce an added direction $y$ such that $[\hat{x}, y] \in \mathbb{R}^{n+1}$ and we define a mapping such that

$y_{N+1}=y_{N}+f\left(\hat{x}_{N}\right)$.

\footnotetext{
${ }^{1}$ Actually, Taylor found that Eq. (1) is only valid for $t \gg$ $0.07 a^{2} / D$ as this is the average time necessary for a tracer particle to sample all locations in the cross-section; for shorter times, convective effects dominate and $\sigma^{2}$ grows as $t^{2}$. A related constraint is that the cross-section must be bounded; we will later examine the consequence of a process whose cross-section is unbounded.
} 
Table 1

Anticipated form of $\sigma_{y}^{2}$ for different systems

\begin{tabular}{lll}
\hline $\begin{array}{l}\text { Nature of system to be investigated } \\
\text { as } N \rightarrow \infty,\end{array}$ & Example & $\begin{array}{l}\text { Behavior of } \sigma_{y}^{2} \text { as } N \rightarrow \infty \\
\hat{x}_{N+1}=y_{N}+f\left(\hat{x}_{N}\right)\end{array}$ \\
\hline $\begin{array}{l}\text { Single attractive fixed point } \\
\text { Single attractive limit cycle }\end{array}$ & Logistic equation with $\lambda<3$ & Bounded \\
Multiple fixed points & Logistic equation with $\lambda>3$ and $\lambda<3.57$ & $\begin{array}{l}\text { Bounded } \\
\sigma_{y}^{2} \text { grows as } N^{2}\end{array}$ \\
$\begin{array}{l}\text { Multiple attractive limit cycles } \\
\text { Topologically transitive but without }\end{array}$ & Twisting map for irrational values of $\alpha$ & $\sigma_{y}^{2}$ grows as $N^{2}$ \\
mixing (non-chaotic) & Trigonometric map & $\sigma_{y}^{2}$ grows as $N^{2}$ \\
Chaotic & & \\
& & $\sigma_{y}^{2}$ grows as $N^{\alpha}, \alpha \neq 2$. \\
$\alpha=1:$ diffusive; & $\alpha \neq 1,0<\alpha<2:$ anomalous diffusion; \\
& & $\alpha>2:$ diffusive in unbounded domain \\
\hline
\end{tabular}

In this table, $f$ is assumed to be a bounded function that is not uniform throught the domain of $\hat{x}$. As noted in the text, it is possible in cases where $\sigma_{y}^{2}$ is expected to grow as $c^{*} N^{\alpha}$ that the constant $c$ is zero; in those cases, the behavior is non-determinant and another function $f$ is chosen.

The coordinate $y$ is analogous to the streamwise direction in the Taylor dispersion problem. Note that displacements in the $y$-direction depend only on the particle location, $\hat{x}$, as determined by the original process being investigated. Thus, no additional dynamics are added to the process by the function $f$.

In the Taylor dispersion problem, the function $f$ is the axial velocity normal to the cross-sectional plane. In that problem, the function $f$ is restricted to be time-independent, or at least statistically steady (for turbulent flows) [15]; it is also required that the axial flow not be uniform within the domain of interest. Otherwise, diffusive behavior in the axial diraction will not be seen.

For chaotic processes, no theoretical analysis is available to indicate the requirement for the function $f$. This would be a useful topic for further research in this area. We have found that, in practice, most choices for $f$ are acceptable. The only restriction is a practical one that the function chosen must generate dispersion in the $y$-direction that increases with the step number $N$, and this is a straightforward proposition to determine. In practice, we allow $f$ to be a linear function of $\hat{x}$ and measure the rate of change of the variance in the $y$-direction. If the variance does not grow with $N$, another function $f$ is chosen. We have found that a linear function almost always suffices, but we will show one example in which a linear function does not generate an increasing dispersion; for that case, we then allow $f$ to be quadratic. We do not investigate, in this paper, the theoretical requirements for the function $f$.

We introduce tracer particles (dispersed randomly throughout the domain $\mathbb{R}^{n}$ ) that are then transported by the process defined by Eqs. (2) and (3). By monitoring the growth of the variance of the tracer distribution in the $y$-direction, $\sigma_{y}^{2}$ (an ensemble average), we can evaluate if the process is a diffusive one. Specifically, a process that is diffusive will have the variance of the tracer distribution grow as $N^{\alpha}, \alpha \neq 2$, while a non-diffusive process will have its variance grow as $N^{2}[16]$. The most generic case of a diffusive process will have $\alpha=1$; diffusive cases with $\alpha<2$ and $\alpha \neq$ 1 are referred to as exhibiting "anomalous diffusion". The anticipated form of $\sigma_{y}^{2}$ for different systems is shown in Table 1.

We will find that some processes will show no long-term in $\sigma_{y}^{2}$, which is a non-determinant case that may or may not be random (chaotic). This happens relatively frequently for non-chaotic mappings as a consequence of the simple form of the attractors that typically arise in one-dimensional systems which lead to all particles approaching a single attractor (fixed point or limit cycle), and thus $\sigma_{1}^{2}$ remains bounded. 
In such a case, all functions $f$ (Eq. (3)) will lead to an upperbound for $\sigma_{y}^{2}$. Chaotic processes occasionally (rarely in our experience) can exhibit bounded values of $\sigma_{y}^{2}$ for a particular $f$, but will show $\sigma_{y}^{2}$ to grow with $N$ for most other choices for $f$. A single case is described (the "biological" map described below) where a linear function $f$ leads to bounded values of $\sigma_{y}^{2}$ but a quadratic function shows $\sigma_{y}^{2}$ to grow with $N$.

It is also possible to have a "mixed regime" for near integrable systems in which part of the domain is regular and the remainder is chaotic [16]. Although we do not further consider such processes here, they can be handled by using similar methods to those we describe, but particles must be seeded exclusively in the chaotic domain to see diffusive growth of the variance.

The ensemble-averaged quantities characterizing the process dynamics were computed only after the stable attractor (fixed point, limit cycle or strange attractor) was reached. The particles were initially distributed randomly and uniformly throughout the domain (usually with $0<x_{0}<1$ ). Double-precision was used for all calculations. We began our computations of the parameters for $N>1000$. The effective diffusion coefficient was calculated as

$D_{\mathrm{eff}}=\lim _{N \rightarrow \infty} \frac{\sigma_{y}^{2}(1000+N)-\sigma_{y}^{2}(1000)}{2 N}$.

We compared use of the diffusive criterion for chaos with the two other criteria, namely a positive Lyapunov exponent and the autocorrelation coefficient dropping to zero. The Lyapunov exponent was computed by introduction a small perturbation $\left(\varepsilon=10^{-9}\right)$ to the system state at $N=1000$ and then computing the exponent $\hat{\mu}$ as

$\hat{\mu}=\frac{1}{N}\left\langle\ln \left|x_{1000+N}^{\prime}-x_{1000+N}\right|\right\rangle / \varepsilon$,

where \langle\rangle is an ensemble average and $x_{1000+N}^{\prime}$ is the value of $x$ at step $1000+N$ having been perturbed at step 1000 by $\varepsilon$.

The correlation coefficient was computed as

$$
r=\frac{\left\langle x_{1000+N} x_{1000}\right\rangle-\left\langle x_{1000+N}\right\rangle\left\langle x_{1000}\right\rangle}{\sigma_{x}(1000+N) \sigma_{x}(1000)},
$$

where $\sigma_{x}(m)$ is the standerd deviation of the distribution of $x$ at step $m$. Note that if $r$ should ever return to 1 for any $N>0$, the process cannot be chaotic.

\section{The logistic equation}

We first consider the well-known logistic equation:

$x_{N+1}=\lambda x_{N}\left(1-x_{N}\right)$.

This equation exhibits chaotic behavior when the parameter $\lambda$ is greater than approximately 3.57 [17]

We introduce motion in the added direction $y$ with $f\left(x_{N}\right)=x_{N}-0.5$ :

$y_{N+1}=y_{N}+\left(x_{N}-0.5\right)$,

and then compute the Layapunov exponent, the autocorrelation coefficient and the variance.

For $\lambda<3$ (attractive fixed points), in all cases, the Layapunov exponent is found to be negative, and the correlation coefficient remains at $1 ; \sigma_{y}^{2}$ remained constant since all particles are attracted to the single stable fixed point. Similar behavior is seen for $3<$ $\lambda<3.57$ (attractive orbits), although there is a long time oscillatory behavior of the variance. An example is shown in Figs. 1(A-C) for $\lambda=3.5$. As expected, no growth was seen in the variance once the limit cycle was reached, even if the computation was extended to 10000 steps. Similar behavior was seen if different functions $f$ were chosen (e.g. $f\left(x_{N}\right)=x_{N}^{2}$ ).

For $\lambda>3.57$, the onset of chaos is accompanied by a positive Layapunov exponent, a decreasing correlation coefficient and the variance growing with $N$ in a fashion consistent with diffusive transport $\left(\sigma_{y}^{2} \sim N\right)$. Figs. 1(G-I) show the behavior of the three chaotic measures for $\lambda=3.9$; all three measure are consistent with a chaotic process with a positive Lyapunov exponent, the autocorrelation coefficient dropping to zero and the variance in the $y$-direction growing linearly with $N$. However, for $\lambda=3.61$, a parameter value also recognized to be the chaotic regime, while both the Lyapunov exponent and the growth of the variance in the $y$-direction are consistent with a chaotic process (Figs. 1(D) and (F)), the autocorrelation coefficient does not drop to zero (Fig. 1(E)) even if the 

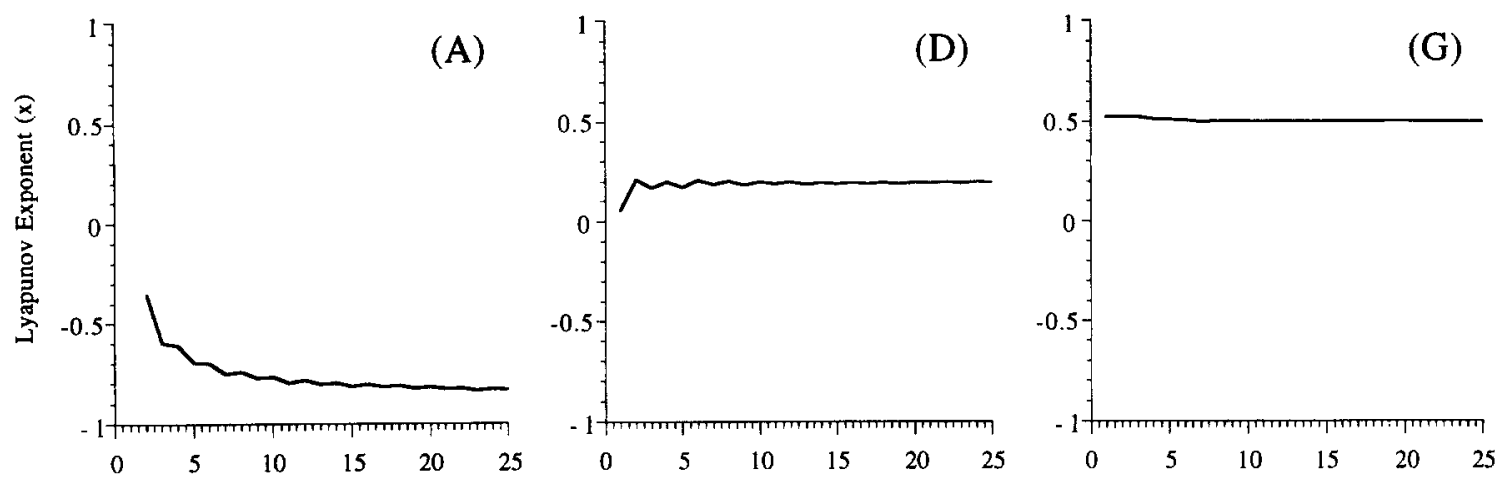

(B)
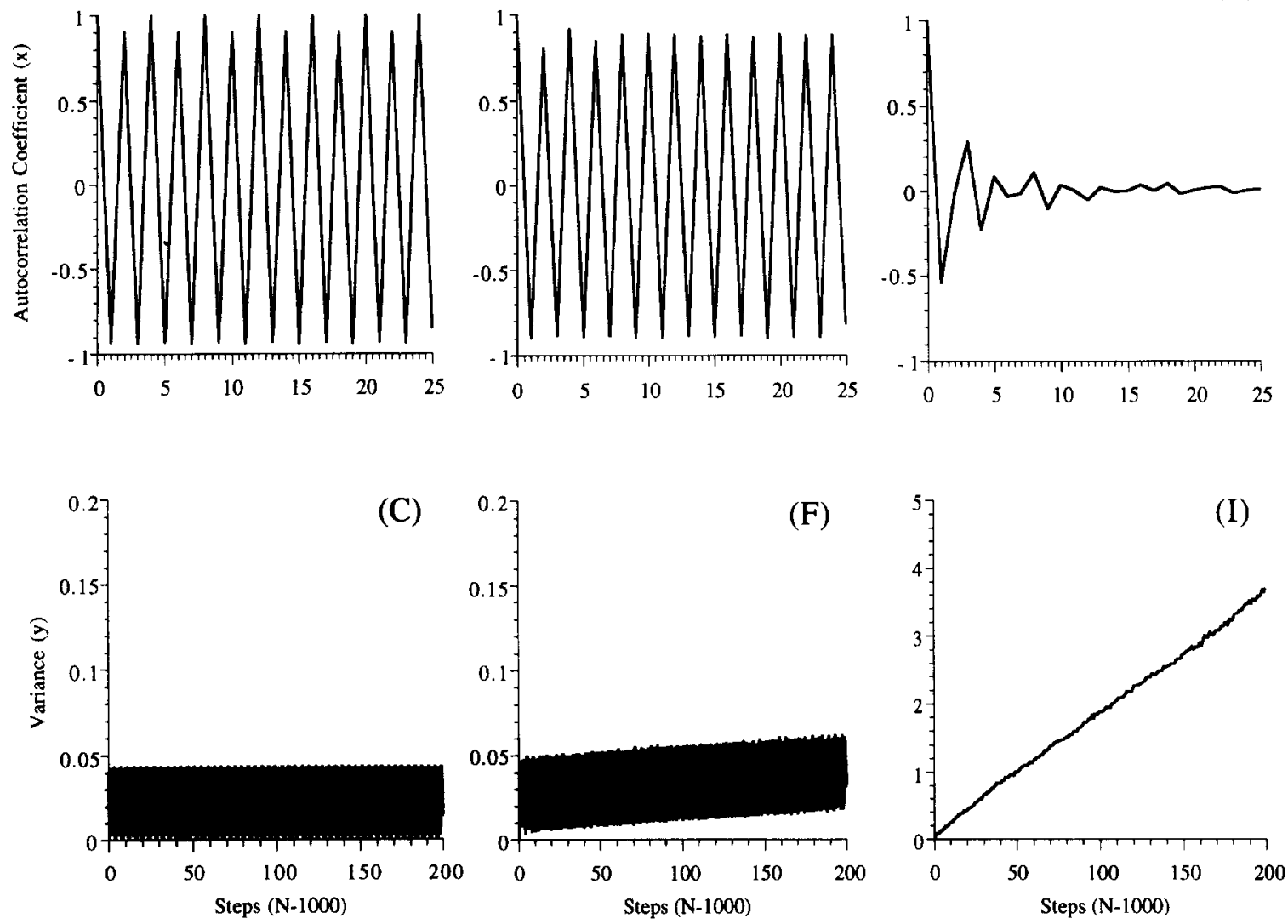

$$
x_{N+1}=3.5 x_{N}\left(1-x_{N}\right) \quad x_{N+1}=3.61 x_{N}\left(1-x_{N}\right)
$$

$$
x_{N+1}=3.9 x_{N}\left(1-x_{N}\right)
$$

Fig. 1. Lyapunov exponent (A, D, G) and autocorrelation coefficient (B, E, H) of $x$ particle location, and variance (C, F, I) of $y$ particle location for three values of $\lambda$ in the logistic equation. Note the change of scale in $I$.

calculations are extended out to $N=10000$ steps. This is an example of a chaotic process which cannot be identified by the criterion of the autocorrelation coefficient dropping to (and remaining at) zero.
To understand how a process can remain autocorrelated for all $N$ (Fig. 1(E)) and yet still be random, consider a general description of the Taylor dispersion process for one-dimensional mapping with $f$ being 
chosen to be linear. Letting $x_{N+1}=g\left(x_{N}\right)$ and $y_{N+1}=y_{N}+\left(x_{N}-a\right)$, we then find that

$$
\begin{aligned}
\sigma_{y}^{2}(N) & =\sum_{i=0}^{N-1} \sum_{j=0}^{N-1}\left\langle\left(x_{i}-\left\langle x_{i}\right\rangle\right)\left(x_{j}-\left\langle x_{j}\right\rangle\right)\right\rangle \\
& =\sum_{i=0}^{N-1} \sum_{j=0}^{N-1} r_{x}(i, j) \sigma_{x}(i) \sigma_{x}(j),
\end{aligned}
$$

where $r_{x}(i, j)$ is the correlation coefficient between the particle's $x$ location at step $i$ and step $j$, and $\sigma_{x}^{2}(i)$ is the variance of the particle's $x$ location at step $i$. (Jones and Young [16] find an analogous expression for a continuous process but use an autocorrelation function that is not normalized.)

Note here that the particularly simple form for $\sigma_{y}^{2}$ results because of the simple form used for $f\left(x_{N}\right)$, i.e. linear. We can then identify those process in which $\sigma_{y}^{2} \sim N$ (i.e. diffusive processes) and we find a number of conditions under which this can occur: (i) $r_{x}(i, i+$ $k$ ) $=0$ for all $k \geq n$ (this occurs for the logistic equation for $\lambda=4$ with $n=1$ ), (ii) $r_{x}(i, i+k) \sim$ $\mathrm{e}^{-k}$, (iii) $r_{x}(i, i+k) \sim k^{-\alpha}$ with $\alpha>1$ (for $0<$ $\alpha \leq 1$, anomalous dispersion [16] results), and (iv) a summation condition on the correlation coefficient:

$\lim _{N \rightarrow \infty}\left|\sum_{k=0}^{N} r_{x}(i, i+k) \sigma_{x}(i) \sigma_{x}(i+k)\right|<S$,

where $S$ is some positive constant. As $\sigma_{x}^{2}(i)$ usually approaches a constant value for large $N$ (i.e. $\sigma_{x}$ becomes roughly $1 / 2$ the domain size), this criterion is equivalent to a requirement that the sum over $N$ (or temporal integral) of the autocorrelation coefficient approaches a constant value for large $N$. This can be achieved either by the autocorrelation coefficient approaching 0 for large $N$, or by a long time oscillation, as seen in the case for $\lambda=3.61$ (Fig. 1(E)). Thus we see that the diffusive criterion for chaos is somewhat less strict than a requirement that the autocorrelation coefficient drops to zero for large $N$.

\section{Other well-known mappings}

We next considered a number of common mappings including: (a) the twisting map $[1,18]$

$$
\theta_{N+1}=\theta_{N}+2 \pi \alpha,
$$

(b) a trigonometric map $[6,19]$

$$
x_{N+1}=0.999 \sin \left(\pi x_{N}\right),
$$

(c) an exponential or "biological" map $[6,20]$

$$
x_{N+1}=x_{N} \exp \left[3\left(1-x_{N}\right)\right],
$$

(d) the $x$ values of the Hénon map [6,21]

$$
x_{N+1}=z_{N}+1-1.4 x_{N}^{2}, \quad z_{N+1}=0.3 x_{N} .
$$

The first of these mappings is not chaotic while the other three are.

For rational values of $\alpha$, the twisting mapping has an infinite number of periodic points; for irrational values of $\alpha$, there are no periodic points. However in neither case is the process topologically transitive (there is no mixing). Thus, this is not a chaotic process.

The particles were seeded initially uniformly between 0 and $2 \pi$. The Lyapunov exponent was found to be zero for all values of $\alpha$. For $\alpha$ rational, the correlation coefficient always returned one (periodic orbits); for $\alpha$ irrational, the correlation coefficient returned arbitrarily close to one. We chose $f\left(\theta_{N}\right)=\theta_{N}$ (linear), substituted into Eq. (3) and used Eq. (11) to find the behavior of $\sigma_{y}^{2}$. We found that for both rational and irrational values of $\alpha, \sigma_{v}^{2}$ varied with $N^{2}$, as expected for a non-random process. For $\alpha$ rational, this growth occurred from one period to the next, while for $\alpha$ irrational, this growth was considerably slower and occurred in an quasi-oscillatory fashion. Thus, all three measures (Lyapunov exponent, correlation coefficient, long-term growth of the variance in the linked coordinate) confirmed that this process was not chaotic.

Turning next to the trigonometric map, the Lyapunov exponent was found to be 0.667 and the correlation coefficient dropped to zero in one step. Using Eqs. (8) and (12) to determine if the process was diffusive, we found $\sigma_{r}^{2}$ to grow linearly with $N$, as expected for a random process. Again, all three measures confirmed that the process was chaotic.

The exponential map also demonstrated a positive Lyapunov exponent $(0.389)$. The correlation coefficient dropped to zero, but in an oscillatory fashion, 


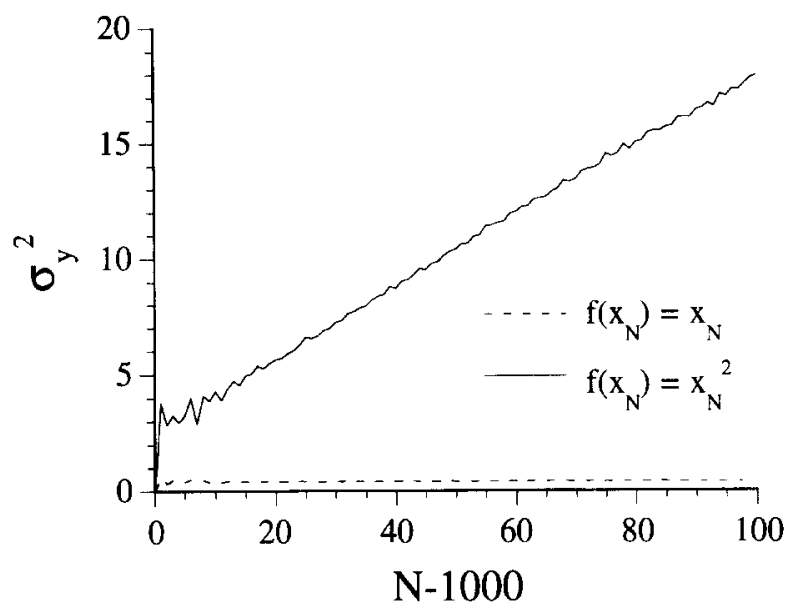

Fig. 2. Variance of the particle location in the added direction $y$ for the exponential map (Eq. (13)).

taking about 20 steps (similar to Fig. 1(H)). Using $f\left(x_{N}\right)=x_{N}$ and Eq. (13) to determine if the process was diffusive, $\sigma_{y}^{2}$ was found to exhibit no long-term growth (see Fig. 2) even if calculations were extended out to 10000 steps; this can be shown to be a consequence of a similarity between the functional form of $f$ (linear) and the solution to the difference equation (13) (see Appendix A). This is an example of a chaotic process that exhibits non-determinant behavior using the growth of the variance in the linked coordinate as a measure. However, when $f\left(x_{N}\right)=x_{N}^{2}$ was used in Eq. (3), $\sigma_{v}^{2}$ showed the linear growth with $N$ as is characteristic of a random process (Fig. 2). Thus, all three measures again agreed that the process was chaotic.

Finally, we examined the Hénon mapping, a twodimensional chaotic mapping. The particles were seeded uniformly in the region $0<x_{0}<1$ with $z_{0}=0$. Again, we found an agreement between the three characterizations of chaos. The Lyapunov exponent (in this case, measured as the $x$-growth of the perturbation) was found to be 0.419 . The $x$ location correlation coefficient dropped to zero in an oscillatory fashion in approximately 15 steps. To investigate the diffusive characteristics of the process, we set $f\left(x_{N}, z_{N}\right)=x_{N}$. Introducing this function into Eq. (3) and using Eq. (14), $\sigma_{y}^{2}$ showed the linear growth characteristic of a random process.
Thus, the four mappings investigated in this section, one non-chaotic and three chaotic, all were characterized correctly by the three measures used: positive Lyapunov exponent, correlation coefficient dropping to zero, and variance of the particle location in the linked corrdinate direction $y$ growing in a diffusive fashion.

\section{Mappings with similar structure to the logistic equation}

The solution to Eq. (7) with $\lambda=4$ is known to be of the form:

$x_{N}=\frac{1}{2}\left\{1-\cos \left[h(N) \cos ^{-1}\left(1-2 x_{0}\right)\right]\right\}$.

with $h(N)=2^{N}$. This equation shows the well-known features that lead to chaos, namely a stretching that results from the function $h(N)$ and a folding that results from the trignometric function. The analogy has been made to bread-making, which involves stretching and folding of the dough [7]. We were curious as to whether the stretching function needed to be exponential as a necessary condition for chaos (i.e. whether a positive Lyapunov exponent was necessary). We examined two further functions, $h(N)=N+1$ and $h(N)=N^{2}+1$, that did not exhibit this exponential growth.

By using the transformation $\theta=1-\cos ^{-1}(1-2 x)$, Eq. (15) can be shown to be semi-conjugate to the circular mapping $\theta_{N+1}=h(N) \theta_{0}$ [1]. Then the three cases considered, $h(N)=2^{N}, N+1$ and $N^{2}+1$, can be shown to correspond to the difference equations $\theta_{N+1}=2 \theta_{N}, \theta_{N+1}=\left(\left(N^{2}+2 N+2\right) /\left(N^{2}+\right.\right.$ 1) $) \theta_{N}$ and $\theta_{N+1}=((N+2) /(N+1)) \theta_{N}$, respectively. $h(N)=2^{N}$ has already been shown to chaotic [1]. $h(N)=N+1$ can be shown to be chaotic by recognizing that (i) the mapping is sensitive to initial conditions (by design), and (ii) it has a dense set of periodic points. Therefore, given that any two of Devaney's three conditions represent a sufficient condition for chaos [2-4], this system is chaotic. $h(N)=$ $N^{2}+1$ can be shown to be chaotic in a similar fashion, although, in this case, it is easier to demonstrate 
that the system is topologically transitive rather than showing it to have dense periodic points.

We proceeded to investigate how the three different indicators of chaos we have used behave for these three mappings. Using Eq. (8) to characterize transport in the linked coordinate, we found that using Eq. (15) with $h(N)=2^{N}$ gave an agreement between all three indicators of chaos. This was, of course, expected since we previously investigated the logistic equation as described in Section 3.

However, a different result was obtained using $h(N)=N+1$ and $N^{2}+1$. Clearly, neither of these functions will give a positive Lyapunov exponent when substituted into Eq. (15), and our numerical results confirmed this. Yet measurement of the correlation coefficient and the growth of the variance in the linked coordinate (using Eq. (8) to characterize transport in this direction) showed both processes to be chaotic, in agreement with Devaney's definition. This was especially surprising for $h(N)=N+1$ as the growth of the error is only linear. (It should be noted that this latter case is particularly interesting in that for an initial condition of uniform probability between $x=0$ and $x=1$, this process exhibits a very unusual form of anomalous diffusion with $\sigma_{y}^{2}$ growing only as $\ln (N)$.)

Thus, we here have demonstrated that chaotic processes do not necessarily have a positive Lyapunov exponent. The diffusive measure of chaos seems to be a more robust determinant.

\section{Diffusion}

We next examined a chaotic process that has already been shown to be positive:

$x_{N+1}=x_{N}-\mu \sin \left(2 \pi x_{N}\right)$.

This process shows the normal period doubling route to chaos, with an accumulation point occurring at approximately $\mu>0.57$. Geisel [10] and Geisel and Nierwetberg [22] showed a change in character in the process when $\mu>0.732644$. For $\mu$ less than this value, the system is still chaotic, but does not exhibit diffusional behavior in the $x$-direction, since the mapping traps the orbits between values of -0.5 and 0.5 ; above this value of $\mu$, the system exhibits diffusion in the $x$-direction with a typical Gaussian distribution. We hypothesized that the only reason diffusion was not observed below this critical value of $\mu$ was that the orbits were trapped to $x$ values between $-1 / 2$ and $1 / 2$, but that the motions were still diffusive. Thus, we introduced $f\left(x_{N}\right)=x_{N}$ and used Eqs. (3) and (16) to investigate whether diffusive motion was occurring.

For this case, a different behavior is expected in terms of the growth of the variance with increasing $N$. For $0.57<\mu<0.732644$, linear growth of $\sigma_{y}^{2}$ with $N$ is expected; however, for $\mu>0.732644$, the orbits in the $x$-direction are not bounded. The Taylor dispersion process shows linear growth of $\sigma_{y}^{2}$ with $N$ only when the orbits in the original domain $\left(\mathbb{R}^{n}\right)$ are bounded. However, it has been shown [23] that for Taylor dispersion in an unbounded shear flow with constant velocity gradient $\mathrm{d} V_{y} / \mathrm{d} x$, the effective diffusion coefficient ( $\left.D_{\text {eff }}\right)$ can be related to the molecular diffusion coefficient $(D)$ as

$D_{\text {eff }} / D=\left(\mathrm{d} V_{y} / \mathrm{d} x\right)^{2} t^{2}$.

Note that in this case, $\sigma_{y}^{2}$ grows with $t^{3}$ and $D_{\text {eff }}$ grows with $t^{2}$ rather than reaching an asymptotic value (a convective, non-chaotic process would show $\sigma_{y}^{2}$ growing with $t^{2}$ whether or not the cross-section was bounded). For $y_{N+1}=y_{N}+x_{N}$, the time step is unity as is the velocity gradient, and thus this relationship becomes

$D_{\text {eff }} / D_{\text {chaos }}=N^{2}$,

where $D_{\text {chaos }}$ is the diffusion coefficient inherent in the chaotic process as previously determined by Geisel.

As expected, we found that diffusive motion (variance growing linearly with $N$ in the linked coorrdinate $y$ ) began for values of $\mu>0.57$, which the correlation coefficient and Lyapunov exponent confirmed as the value at which chaos began. This supported our hypothesis that diffusive motion was apparent for all chaotic values of $\mu$. We further investigated this phenomenon by examining the relationship between the diffusion coefficient $D_{\text {chaos }}$ measured in the $x$ direction $\left(D_{\text {chaos }}=1 / 2 \mathrm{~d} \sigma_{x}^{2} / \mathrm{d} x\right)$ and the value of 


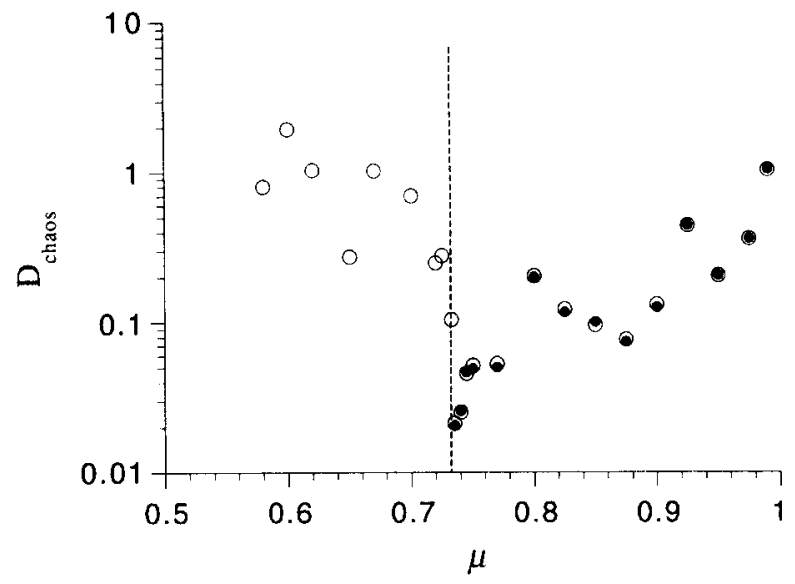

Fig. 3. Diffusion coefficient as a function of $\mu$ for $x_{N+1}=x_{N}-\mu \sin \left(2 \pi x_{N}\right)$. Open circles show values calculated using Taylor dispersion in $y$-direction; filled circles are calculated directly from growth of second moment in $x$-direction. The vertical dashed line is at $\mu=0.732644$. No filled circles are given for $\mu<0.732644$, since, as explained in the text, the orbits are trapped below this value of $\mu$ and thus direct calculation of the diffusion in the $x$-direction cannot be accomplished.

$D_{\text {chaos }}$ determined from Eqs. (4) and (18). As shown in Fig. 3, an excellent agreement was seen between the two methods of calculation for $\mu>0.732644$.

For $0.57<\mu<0.732644$, as mentioned previously, no direct measure of the diffusion coefficient $\left(D_{\text {chaos }}\right)$ was possible. However, we used the analytical result for Taylor dispersion in a Couette flow (see Eq. (20), below, with $D_{\text {chaos }}$ in place of $D_{\text {intrinsic }}$ ) to estimate values of $D_{\text {chaos }}$, as the orbits were constrained in this case to be between $-1 / 2$ and $1 / 2$. This procedure yielded values of similar order of magnitude to those found for $\mu>0.732644$.

\section{Effect of added intrinsic diffusion}

For the chaotic processes investigated, we have computed the values of the effective diffusion coefficient $\left(D_{\text {eff }}\right)$ in the linked coordinate $y$ (Eq. (4)). The question arises as to whether the magnitude of this quantity gives additional information concerning the nature of the chaotic processes being investigated. In particular, does greater chaotic mixing lead to a greater or less values of $D_{\text {eff }}$ ? Eq. (1) would suggest the latter, but it must be recalled that $D_{\text {eff }}$ depends not

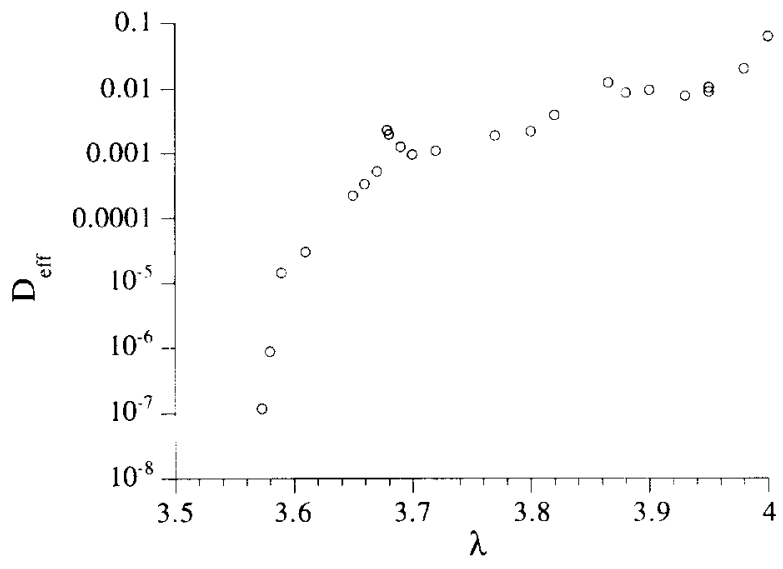

Fig. 4. Effective diffusion coefficient in the linked coordinate $y$ as a function of $\lambda$ as determined using Eqs. (4). (7) and (8).

only on the level of cross-sectional mixing, but also on the area over which this mixing occurs. As the area (measured in the appropriate fractal dimension) of the strange attractor will change for different chaotic conditions, it is not clear how $D_{\text {eff }}$ is quantitatively related to the extent of the chaotic mixing. An example is given in Fig. 4 showing the effect of increasing $\lambda$ on $D_{\text {eff }}$ for Eqs. (7) and (8) as determined in Section 3.

To further investigate this, we added intrinsic diffusion, or "noise", to Eq. (7) following the method of Crutchfield and Farmer [17], and determined the effect of the intrinsic diffusivity on $D_{\text {eff }}$. We added the random variable $s$ to $\mathrm{Eq}$. (7) to yield

$x_{N+1}=\lambda x_{N}\left(1-x_{N}\right)+s$.

$s$ was chosen using a random number generator with a standard deviation $\sigma_{s}^{2}=2^{*} D_{\text {intrinsic }}$ and zero mean. If, due to $s$, Eq. (19) yielded a value that was outside of the domain $0 \leq x_{N+1} \leq 1$, the value was reflected back into the domain.

Fig. 5 shows results for $D_{\text {eff }}$ as a function of $D_{\text {intrinsic }}$ for $\lambda=4$ and $\lambda=3.61$. Also shown is the classical Taylor dispersion result which is found when $x_{N+1}=x_{N}+s$ (only intrinsic diffusion, no chaotic motion). In the latter case, the Taylor dispersion result (Couette flow) is found to be

$D_{\text {eff }}=\frac{2 a^{2} V_{\max }^{2}}{15 D_{\text {intrinsic }}}$, 


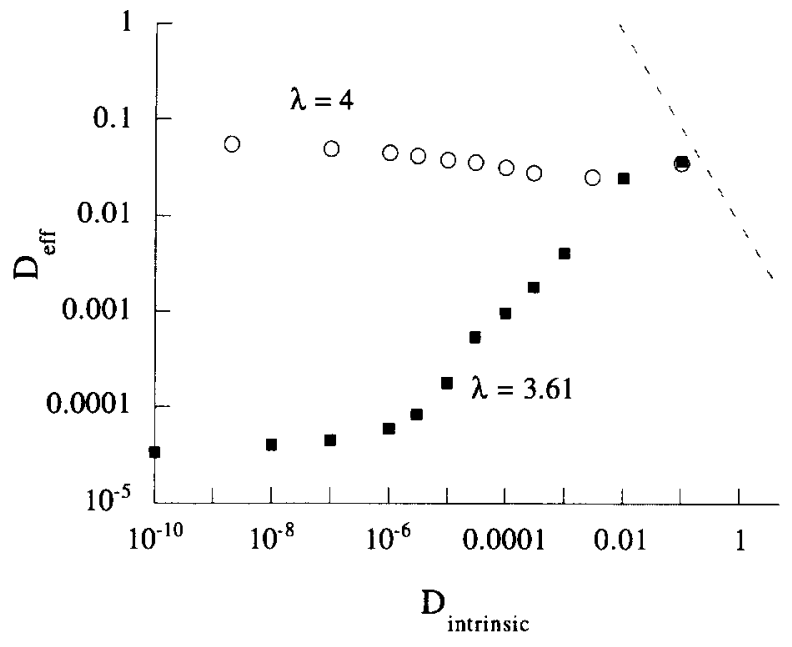

Fig. 5. $D_{\text {eff }}$ for Eq. (19) as a function of $D_{\text {intrinsic }}$ for $\lambda=3.61$ (squares), $\lambda=4$ (circles), and for Eq. (20) (dashed line).

with $2^{*} a$ the domain size and $V_{\max }$ the maximum axial velocity in the domain. For the given conditions $\left(V_{\max }=1 / 2 ; a=1 / 2\right), D_{\text {eff }}=0.00833 / D_{\text {intrinsic }}$.

While the results for $\lambda=4$ show that increasing $D_{\text {intrinsic }}$ leads to a decrease in $D_{\text {eff }}$, as might be anticipated from Eq. (1), $\lambda=3.61$ shows a different behavior; here the converse occurs. (Note that both values of $\lambda$ approach the classical Taylor result for high values of $D_{\text {intrinsic }}$, as one would expected). The different behavior of $\lambda=4$ and $\lambda=3.61$ is due to the change in character of the strange attractor as $D_{\text {intrinsic }}$ is increased.

While $\lambda=4$ is associated with strange attractor that spans the entire domain $0 \leq x_{N} \leq 1, \lambda=3.61$ has a much smaller attractor that is concentrated in two regions (roughly $0.31 \leq x_{N} \leq 0.62$ and $0.78 \leq x_{N} \leq$ 0.91 ). Thus, when intrinsic diffusion is added to the latter case, it increases not only the random mixing on the strange attractor, but also expands its size, ultimately, for high values of $D_{\text {intrinsic }}$, filling the domain, $0 \leq x_{N} \leq 1$. This, of course, affects the magnitude of $D_{\text {eff }}$ and shows the difficulty of characterizing the extent of chaotic mixing using $D_{\text {eff }}$, since it characterizes both the extent of mixing and the region over which mixing occurs. Thus, while we have found $D_{\text {eff }}$ to be a useful tool for determining whether or not a given process is chaotic, we cannot yet assign physical significance to its magnitude.

\section{Discussion and conclusions}

Deterministic processes that exhibit stochastic behavior are characterized as "chaotic" if they have a low number of degrees of freedom and "random" if they have a high number of degrees of freedom [6]. This characterization is not well-defined since there is no sharp boundary between these behaviors. However, the hallmark and surprising characteristic of all these deterministic processes is their intrinsic stochastic behavior. Since stochastic behavior is invariably associated with diffusion, we have devised a test that allows any system to be probed for diffusive character.

Our new operational test for chaos appears consistent with the definition of chaotic processes, at least for discrete mappings. Although this test does not distinguish between low-order and high-order chaotic systems, other tests already exist to examine that distinction [6]. While we have examined discrete mapppings, other investigators have used an analogous scheme to ours for investigating continuous systems $[16,24,25]$. These have been flow processes in which chaotic behavior in the cross-sectional plane has been demonstrated to lead to diffusive or anomalous diffusive behavior in the axial direction, adding support to the content that all chaotic processes exhibit diffusive behavior. However, we have not yet demonstrated theoretically that Devaney's definition of chaos [1], in fact, requires diffusive behavior.

We have further shown that two other frequently used criteria for chaos, a positive Lyapunov exponent and an autocorrelation coefficient dropping to and remaining at zero, fail to be satisfied for some mappings that meet Devaney's definition of chaos. In the case of positive Lyapunov exponent, its requirement for exponential growth appears to be too strong. Devaney's definition only requires sensitivity to initial conditions. As regards the autocorrelation coefficient, we have shown that diffusive growth of the variance in the added direction can be related to the autocorrelation coefficient (Eq. (10)). This relationship indicates that diffusive behavior is associated with the sum (or integral) of the autocorrelation coefficient rather than its value at any single step. 
In summary, we have presented a new technique for detecting the stochastic behavior characteristic of chaotic process. In all the cases examined, the technique has correctly identified chaotic behavior. Further theoretical studies are necessary to find if Devaney's definition of chaos necessarily implies stochastic behavior.

\section{Acknowledgements}

We thank the MIT Undergraduate Research Opportunities Program for providing support for this project. We also thank Professors Anthony Patera, Roger Kamm and Zaichun Feng for helpful discussions.

\section{Appendix A}

As mentioned in Section 4, use of a linear function for $f\left(x_{N}\right)$ in Eq. (3) combined with the exponential map (13) leads to no long-term growth for $\sigma_{y}^{2}$. We here demonstrate why that particular result arises. Beginning with Eq. (13), we can solve this difference equation to find that

$x_{N+1}=x_{0} \prod_{i=0}^{N} \mathrm{e}^{3\left(1-x_{i}\right)}$

Eqs. (3) and (A.1) can be combined to find the motion in the added direction $y$, as

$y_{N+1}=\sum_{j=0}^{N} x_{j}=\sum_{j=0}^{N} x_{0} \prod_{i=0}^{j-1} \mathrm{e}^{3\left(1-x_{i}\right)}$

or

$$
\begin{aligned}
y_{N+1} & =x_{0} \sum_{j=0}^{N} \mathrm{e}^{\sum_{i=0}^{j-1} 3\left(1-x_{i}\right)} \\
& =x_{0} \sum_{j=0}^{N} \mathrm{e}^{3\left(j-y_{j}\right)}
\end{aligned}
$$

Now introducing $Z_{N} \equiv y_{N}-N-\ln x_{0}$, we find that $Z_{N+1}-Z_{N}=\mathrm{e}^{-3 Z_{n}}-1$.

Note that Eq. (A.4) indicates that growth of orbits in the $y$-direction are limited around the mean position, and thus the variance in this direction cannot continue to grow without bound. This limitation arises because of a similarity between the functional form of $f$ and the solution of the difference equation (A.1). However, if $f$ is chosen as quadratic, the step leading to Eq. (A.3) is not possible, and this limitation in orbit does not occur.

\section{References}

(1) Devaney, An Introduction to Chaotic Dynamical Systems, Addison-Wesley, Reading, MA, 1989.

[2] E. Glasner, B. West, Sensitive dependence on initial conditions, Nonlinearity 6 (1993) 1067-1075.

[3] J. Banks, J. Brooks, G. Cairns, G. Davies, P. Stacey, On Devaney's definition of chaos, Amer. Math. Month. 99 (1992) 332-334.

[4] S. Silverman, On maps with dense orbits and the definition of chaos, Rocky Mountain J. Math. 22 (1992) 353-375.

[5] T.-Y. Li. J.A. Yorke, Period three implies chaos. Amer. Math. Month. 82 (1975) 985-992.

[6] W. Marzocchi, Detecting low-dimensional chaos in time series of finite length generated from discrete parameter processes, Physica D 90 (1996) 31-39

[7] M. Toda. R. Kubo, N. Saitô. Statistical Physics I. Equilibrium Statistical Mechanics, Springer, Berlin. 1983. pp. 206-208.

18| A. Wolf, J.B. Swift, H.L. Swinney, J.A. Vasatano, Determining Lyapunov exponents from a time series. Physica D 16 (1985) 285-317.

19| S. Grossman, S. Thomae, Invariant distributions and stationary correlation function of one-dimensional discrete processes. Zeit Naturforschung, A 32 (1977) 1353-1363.

[10] T. Geisel, Deterministic diffusion - a quality of chaos, in: M.G. Velarde (Eds.), Nonequilibrium Cooperative Phenomenon and Related Fields. Plenum Press. New York, 1983, pp. $437-453$.

[11] A.J. Lichtenberg, M.A. Lieberman, in: Regular and Stochastic Motion, Chapter 5, Springer, New York, 1983.

[12] A.N. Yannacopoulos, G. Rowlands, Calculation of diffusion coefticients for chaotic maps, Physica D 65 (1993) 71-85.

[13] B. Andersson. T. Berglin, Dispersion in laminar flow through a circular tube, Proc. Roy. Soc. London. A 377 (1981) 251-268.

[14] G.I. Taylor. Dispersion of soluble matter in solvent flowing slowly through a tube, Proc. Roy. Soc. London A 219 (1953) 186-203.

[15] P.C. Chatwin, C.M. Allen, Mathematical models of dispersion in rivers and estuaries. Annu. Rev. Fluid Mech. 17 (1985) 119-149.

[16] S.W. Jones. W.R. Young, Shear dispersion and anomalous diffusion by chaotic advection, J. Fluid Mech. 280 (1994) $149-172$. 
[17] J.P. Crutchfield, J.D. Farmer, Fluctuations and simple chaotic dynamics, Phys. Rep. (Rev. Sect. Phys. Lett.) 92 (1982) 45-82.

[18] J.M. Ottino, The Kinematics of Mixing: Stretching, Chaos and Transport, Cambridge University Press, Cambridge, 1989.

[19] N. Metropolis, M.L. Stein, P.R. Stein, On finite limit sets for transformation on the unit interval, J. Combin. Theory 15 (1973) 25-44.

[20] R.M. May, G.F. Oster, Bifurcations and dynamic complexity in simple ecological models, Amer. Natur., 110 (1976) 573-617.

[21] M. Hénon, A two-dimensional mapping with a strange attractor, Commun. Math. Phys. 50 (1976) 69-77.

[22] T. Geisel, J. Nierwetberg, Self-generated diffusion and universal critical properties in chaotic systems, in: Dynamical Systems and Chaos, Lecture Notes in Physics, vol. 179, 1982, Springer, Berlin, pp. 93-114.

[23] R.T. Foister, G.M. Van de Ven, Diffusion of brownian particles in shear flows, J. Fluid Mech. 96 (1980) 105-132.

[24] S.W. Jones, Dispersion in zero-mean flows, Phys. Fluids 7 (1995) 898-900.

[25] V. Ganesan, M.D. Bryden, H. Brenner, Chaotic heat transfer enhancement in rotating eccentric annular-flow systems, Phys. Fluids (1997), in press. 